\title{
A case of Bickerstaff brainstem encephalitis successfully treated with intravenous immunoglobulin and methylprednisolone after unsuccessful immunoadsorption plasmapheresis
}

\author{
Takeshi Imai, Takahiro Shimizu, Yuta Hagiwara, Toshikazu Hirayama, Yasuhiro Hasegawa
}

Department of Internal Medicine, Division of Neurology, St. Marianna University School of Medicine, Kawasaki, Japan Email: t2imai@marianna-u.ac.jp

Received 1 January 2013; revised 8 February 2013; accepted 19 February 2013

\begin{abstract}
Bickerstaff brainstem encephalitis (BBE) is a rare post-infectious neurological syndrome for which an effective treatment strategy has not been established. Here, we report a case of a 71-year-old male who suffered from an upper respiratory tract infection, and 7 days later, developed numbness of the bilateral upper and lower limbs, unsteady gait and dysarthria. Brain magnetic resonance imaging was normal, nerve conduction study and cerebral spinal fluid analysis were nonspecific. Based on the clinical features, we tentatively diagnosed Guillain-Barré syndrome and started immunoadsorption plasmapheresis. However, consciousness progressively declined to coma level within 10 days. Electroencephalogram showed diffuse slowing, and auditory evoked brainstem response (ABR) demonstrated absence of waves II, III and V. Serum anti-GQ1b IgG autoantibody and anti-GM1b IgG autoantibody were negative. Subsequently, we diagnosed BBE, and clinical symptoms resolved after treatment with intravenous immunoglobulin and methyllprednisolone. On day 62, neurological symptoms were remarkably alleviated with an improvement in ABR. Our observations suggest that immunoadsorption plasmapheresis should be used only when antiganglioside antibodies are detected. Combination therapy with intravenous immunoglobulin and methylprednisolone or plasma exchange are recommended as initial therapy.
\end{abstract}

Keywords: Bickerstaff Brainstem Encephalitis; Intravenous Immunoglobulin; Methylprednisolone; Immunoadsorption Plasmapheresis

\section{INTRODUCTION}

Bickerstaff brainstem encephalitis (BBE), first described in 1951, is a rare post-infectious neurological syndrome characterized by ophthalmoplegia, ataxia and symptoms suggesting central nervous system involvement, such as impaired consciousness, extensor plantar responses and hemisensory loss [1]. BBE shares common features with Miller Fisher syndrome (MFS), both clinically and immunologically, and accumulating evidence suggests that BBE and MFS are part of a continuous spectrum of variable central and peripheral nervous system involvement [2]. However, the nosological relationship of BBE to MFS or Guillain-Barré syndrome (GBS) remains controversial, and effective therapy for BBE has yet to be established. Various treatments have been reported for BBE, such as 1) a combination of steroids and plasma exchange, 2) various types of plasmapheresis without steroids, 3) steroids only, 4) a combination of steroids and intravenous immunoglobulin (IVIg) and plasma exchange, and 5) nonspecific immunotherapy [3]. In this report, we describe a patient with anti-GQ1b antibodynegative BBE who was successfully treated with IVIg and methylprednisolone.

\section{CASE REPORT}

A 71-year-old man noticed numbness of both lower limbs 10 days after an upper respiratory tract infection. Within the next two days, he developed numbness of both upper limbs and was unable to walk without assistance. He was admitted to our hospital 2 weeks later because of a progression of motor and sensory disturbances and dyspnea. On admission, consciousness was lucid and eye movement was normal, but bilateral peripheral facial nerve palsy and dysarthria were observed. Using manual muscle testing (MMT), trace contraction was not demonstrated in the upper or lower limbs (MMT 0), and gloveand-stocking type hypoesthesia was observed. Deep tendon reflexes were hyporeflexic, jaw reflex was negative, and Babinski's sign was not observed. His relatives re- 
ported neither toxin intake nor alcohol or drug consumption. As past history, he was diagnosed with atrial fibrillation 10 years earlier. Blood pressure was 125/82 mmHg and heart rate was $70 / \mathrm{min}$. Hematological, hepatic, renal and heart function parameters were normal. He was intubated and placed on a ventilator because of respiratory failure. Lumbar puncture, performed on day 1, yielded clear cerebral spinal fluid (CSF), with zero white blood cells, $83 \mathrm{mg} / \mathrm{dl}$ of protein (normal value $<45 \mathrm{mg} / \mathrm{dl}$ ) and $84 \mathrm{mg} / \mathrm{dl}$ of glucose. Oligoclonal banding was negative in CSF. Brain magnetic resonance imaging (MRI) was normal. We diagnosed the patient as having GBS, and three sessions of IAPP were performed on days 1, 3 and 6. IAPP therapy was conducted using a tryptophan-immobilized column (TR-350; Asahi Medical Co., Tokyo). Tests for IgG antibodies against GQ1b, GM1, GM2, GD1a and GD1b, measured by enzyme-linked immunosorbent assay (ELISA), were negative. Blood and CSF cultures were negative, and serological tests for cytomegalovirus, Epstein-Barr virus and herpes simplex virus were negative. Nerve conduction study on day 3 showed normal CMAP amplitudes with delayed motor and sensory conduction velocities in the median, ulnar, sural and tibial nerves. Distal median sensory nerve conduction velocities were not measurable.

The clinical course of this patient is shown in Figure 1. After two sessions of IAPP treatment, no improvement was observed. On day 4, his consciousness further declined to E1VTM1 (Glasgow coma scale), with bilateral mydriasis and total ophthalmoplegia. Spontaneous breathing was undetectable by the respirator. Muscle tonus was flaccid in both arms and legs. Brain MRI on days 1 and 4 demonstrated no definite lesions, but electroencephalogram revealed bilateral $\theta$ activity, and auditory evoked brainstem response (ABR) showed the ab-

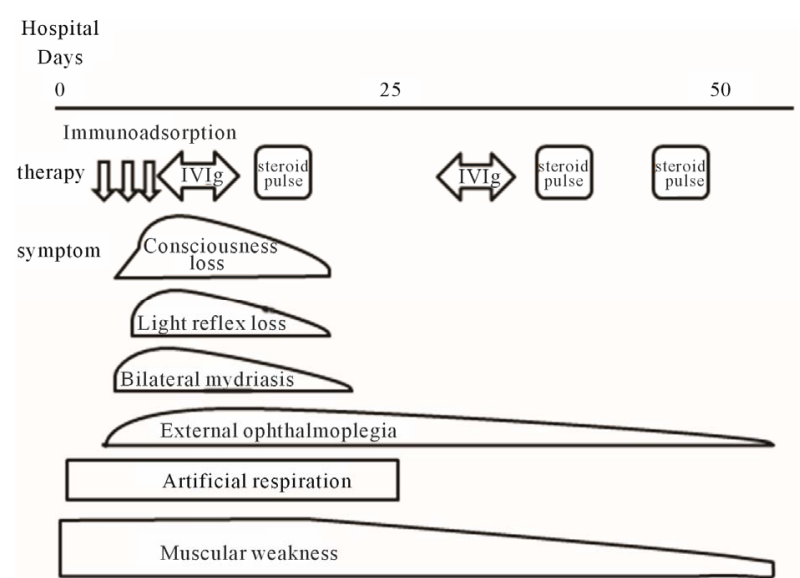

Figure 1. Clinical course. Immunoadsorption: immunoadsorption therapy using the tryptophan-immobilized polyvinyl alcohol gel column (IM-TR 350); IVIg: Venilon-I 0.4 g/kg $\times 5$ days; Steroid pulse: methylprednisolone $1000 \mathrm{mg} /$ day. sence of II, III and V waves on both sides on day 7 (Figure 2). Ultimately, we diagnosed the patient as having BBE. On day 8, human IVIg infusion, $0.4 \mathrm{~g} / \mathrm{kg}$ daily for 5 days, was started. Consciousness gradually recovered, and the pupillary light reflex returned to normal. On day 15, methylprednisolone infusion, $1 \mathrm{~g} /$ daily, was started for three days. Subsequently, respiratory failure began to recover, but tracheostomy was performed because he was still comatose. After IVIg and methylprednisolone treatment, respiratory failure gradually improved to normal breathing without a respirator on day 30. Based on his clinical improvement, IVIg and methylprednisolone treatment appeared very effective. In total, we performed one IVIg session and two sessions of methylprednisolone treatment. On day 62, his ABR showed remarkable improvement (Figure 3). On day 70, he had no neurological deficits apart from mild motor weakness (MMT 3 in both arms and MMT 4 in both legs) and was transferred to a rehabilitation hospital.

\section{DISCUSSION}

BBE can be defined as a syndrome presenting acute ophthalmoplegia, ataxia and disturbance of consciousness as its major manifestations, in association with certain symptoms and signs indicative of central involvement. A considerable number of patients with BBE also develop flaccid tetraparesis, which is presumed to be a sign of overlapping GBS. Brain MRI detects brainstem and cerebellar lesions in only $30 \%$ of BBE patients [4]. Serum anti-GQ1b IgG antibody can be detected in $66 \%$ of BBE patients [4]. In our patient, brain MRI was normal and anti-ganglioside antibodies were negative, but he had antecedent infection, signs of central nervous system involvement and external ophthalmoplegia. In addition, EEG and ABR were abnormal. Thus, the clinical features

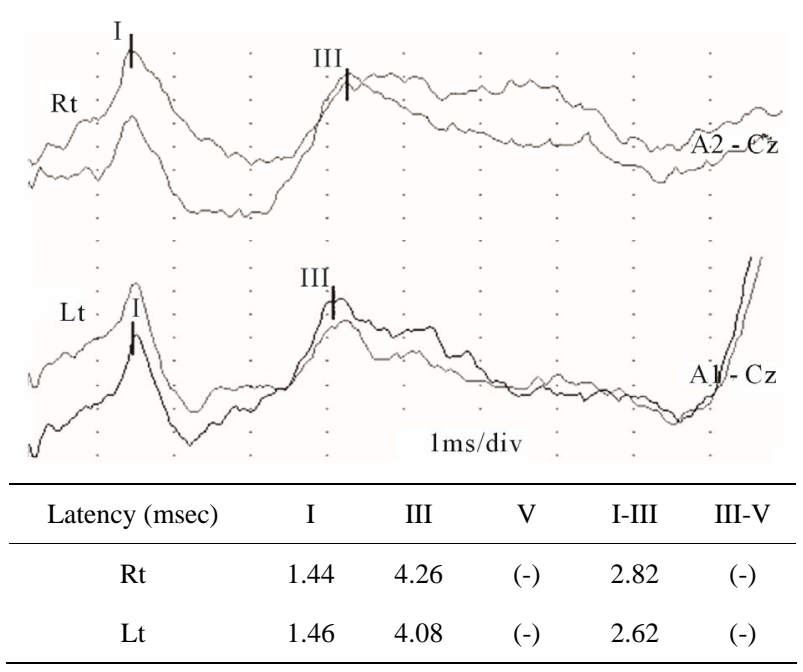

Figure 2. ABR on day 7. ABR showed the absence of II, III and $\mathrm{V}$ waves on both sides. 


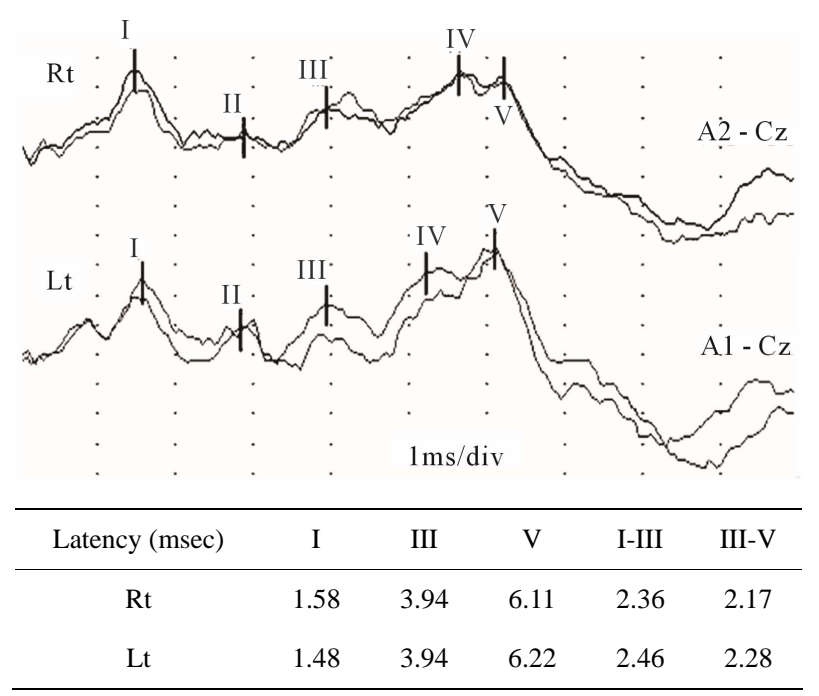

Figure 3. ABR on day 60. ABR showed remarkable improvement.

of this patient were consistent with BBE.

The relatively low prevalence of BBE prevents randomized clinical trials for the determination of effective therapy. At present, IVIg and plasmapheresis (IAPP, double filtration plasmapheresis and $\mathrm{PE}$ ) are the main treatments for BBE, as for GBS and MFS [5]. Several reports have suggested that plasma exchange and IVIg are benefical for BBE [6]. In GBS, there is a consensus that both IVIg and plasma exchange are effective [3,7]. However, a large amount of fresh plasma is needed in plasma exchange therapy, and there is a potential risk of infection and allergy. IAPP is designed to selectively remove the immunoglobulin fraction instead of whole plasma. IAPP has been put forward as an effective treatment for GBS [8]. Treatment with IAPP using a tryptophan-immobilized column was introduced for patients with GBS to remove antibodies to gangliosides. Because we initially diagnosed this patient as having GBS, we commenced IAPP treatment, but his symptoms progressively worsened. The efficacy of IAPP for the treatment of BBE remains controversial. Antibodies to gangliosides can be selectively removed using a tryptophan-immobilized column. However, serum antibodies to gangliosides (anti-GQ1b or anti-GT1a IgG) are undetectable in 33\% of BBE patients [4], suggesting that IAPP may not be effective for anti-ganglioside antibody-negative BBE.

Accumulating evidence suggests that combination therapy with IVIg and high-dose methylprednisolone is more effective than IVIg alone in patients with GBS [9]. If we accept the premise that BBE, GBS and MFS form part of a continuous pathological spectrum with variable central and peripheral nervous system involvement, it might be reasonable to initiate IVIg and methylprednisolone combination therapy for BBE, particularly as BBE may rep- resent the severe end of this spectrum. Combination therapy with IVIg and methylprednisolone is now recommended for patients with BBE by Japanese guidelines [10]. However, randomized controlled trials have not yet been performed, and further studies are necessary to establish a standard treatment for BBE. The mechanisms underlying the efficacy of IVIg in demyelinating disease, such as GBS and BBE, is unclear. The results of two early trials on GBS [11,12] showed no benefit of treatment with low or high-dose prednisolone. Although several mechanisms have been proposed for the beneficial effects of steroids for demyelinating diseases, including the inhibition of inflammatory reactions and decreasing endoneurial pressure and edema, the exact mechanism is unknown [13-15].

IVIg therapy for BBE negative for anti-ganglioside antibodies has been reported by several authors, but the effects varied [16,17]. As a first line treatment for antibody-positive BBE, Tagawa et al. recommended plasma exchange rather than IAPP, because the ability of IAPP to remove anti-ganglioside IgGs and IgMs is inferior to that of plasma exchange [18]. To the best of our knowledge, there is no information on the difference in efficacy of combination therapy for BBE patients with antiganglioside antibodies versus those without. It is reasonable to recommend combination therapy with IVIg and prednisolone or plasma exchange for BBE patients with unknown target antibodies. IAPP should be considered only when anti-ganglioside antibodies are detected. Further studies are necessary to establish a treatment for antiganglioside antibody-negative BBE patients.

\section{REFERENCES}

[1] Bickerstaff, E.R. and Cloake, P.C.P. (1951) Mesencephalitis and rhombencephalitis. British Medical Journal, 2, 77-81. doi:10.1136/bmj.2.4723.77

[2] Ito, M., Kuwabara, S., Odaka, M., Misawa, S., Koga, M., Hirata, K., et al. (2008) Bickerstaff's brainstem encephalitis and Fisher syndrome form a continuous spectrum: Clinical analysis of 581 cases. Journal of Neurology, 255, 674-682. doi:10.1007/s00415-008-0775-0

[3] Overell, J.R., Hsieh, S.T., Odaka, M. and Yuki, N. (2007) Treatment for Fisher syndrome, Bickerstaff's brainstem encephalitis and related disorders. Cochrane Database of Systematic Reviews, 1, CD004761.

[4] Odaka, M., Yuki, N., Yamada, M., Koga, M., Takemi, T., Hirata, K., et al. (2003) Bickerstaff's brainstem encephalitis: Clinical features of 62 cases and a subgroup associated with Guillain-Barré syndrome. Brain, 126, 2279-2290. doi:10.1093/brain/awg233

[5] Tagawa, Y., Yuki, N. and Hirata, K. (1998) Ability to remove immunoglobulins and anti-ganglioside antibodies by plasma exchange, double-filtration plasmapheresis and immunoadsorption. Journal of the Neurological Sciences, 


\section{7, 90-95. doi:10.1016/S0022-510X(98)00067-7}

[6] Yuki, N. (1995) Successful plasmapheresis in Bickerstaff's brain stem encephalitis associated with anti-GQ1b antibody. Journal of the Neurological Sciences, 131, 108110. doi:10.1016/0022-510X(95)00112-F

[7] Mori, M., Kuwabara, S., Fukutake, T. and Hattori, T. (2007) Intravenous immunoglobulin therapy for Miller Fisher syndrome. Neurology, 68, 1144-1146. doi:10.1212/01.wnl.0000258673.31824.61

[8] Hirai, K., Kihara, M., Nakajima, F. and Yoshioka, H. (1998) Immunoadsorption therapy in Guillain-Barré syndrome. Pediatric Neurology, 19, 55-57. doi:10.1016/S0887-8994(98)00010-1

[9] Van Koningsveld, R. and Schmitz, P.I. (2004) Effect of methylprednisolone when added to standard treatment with intravenous immunoglobulin for Guillain-Barré syndrome: Randomized trial. Lancet, 363, 192-196. doi:10.1016/S0140-6736(03)15324-X

[10] Masaaki, O.D.A.K.A. (2008) Bickerstaff's brainstem encephalitis-Continuous spectrum with Fisher syndrome. Neurological Medicine, 69, 5-12

[11] Hughes, R.A., Newsom-Davis, J.M., Perkin, G.D. and Pierce, J.M. (1978) Controlled trial prednisolone in acute polyneuropathy. Lancet, 2, 750-753. doi:10.1016/S0140-6736(78)92644-2

[12] Guillain-Barré Syndrome Steroid Trial Group (1993) Double-blind trial of intravenous methylprednisolone in Guillain-Barré syndrome. Lancet, 341, 586-590.
[13] Kazatchkine, M.D. and Kaveri, S.V. (2001) Immunomodulation of autoimmune and inflammatory diseases with intravenous immune globulin. The New England Journal of Medicine, 345, 747-755. doi:10.1056/NEJMra993360

[14] Berciano, J., Figols, J., García, A., Calle, E., Illa, I. and Lafarga, M. (1997) Fulminant Guillain-Barré syndrome with universal inexcitability of peripheral nerves: A clinicopathological study. Muscle Nerve, 20, 846-857. doi:10.1002/(SICI)1097-4598(199707)20:7<846::AID-M US9>3.0.CO;2-7

[15] Berciano, J. (1998) Rational therapy of Guillain-Barré syndrome. Lancet, 351, 754-755. doi:10.1016/S0140-6736(05)78528-7

[16] Sharma, V., Chan, Y.C., Ong Teoh, H.L. and WilderSmith, E.P. (2006) Bickerstaff's brainstem encephalitis: Can it recur? Journal of Clinical Neuroscience, 13, 277 279. doi:10.1016/j.jocn.2005.01.011

[17] Hussain, A.M., Flint, N.J., Livsey, S.A., Wong, R., Spiers, P. and Bukhari, S.S. (2007) Bickerstaff's brainstem encephalitis related to Campylobacter jejuni gastroenteritis. Journal of Clinical Pathology, 60, 1161-1162. doi:10.1136/jcp.2006.046284

[18] Tagawa, Y., Yuki, N. and Hirata, K. (1998) Ability to remove immunoglobulins and anti-ganglioside antibodies by plasma exchange, double-filtration plasmapheresis and immunoadsorption. Journal of the Neurological Sciences, 157, 90-95. doi:10.1016/S0022-510X(98)00067-7 